\title{
Beitrag zur Polychaetenfauna der Bahia Quillaipe (Süd-Chile)
}

\author{
Gesa Hartmann-Schröder* \\ Biologische Anstalt Helgoland; Notkestr. 31, D-W-2000 Hamburg 52, \\ Bundesrepublik Deutschland \\ und \\ Zoologisches Institut und Zoologisches Museum der Universität Hamburg; \\ Martin-Luther-King-Platz 3, D-W-2000 Hamburg 13, Bundesrepublik Deutschland * *
}

\begin{abstract}
Contribution to the polychaete fauna of Bahia Quillaipe (Chile). 19 species were found; 2 of them are new to science (Scolelepis brevibranchia, Scolelepis crenulata). Additional descriptions of the parapodial lobes of the little-known species Aglaophamus peruana are given. Hemipodus heteropapillatus and Cirriformia quetalmahuensis, described by the author in 1962 as new species, were found again. 2 species could not be determined to species level; one of them, Euclymene (E.) sp., may also be a new species. 4 of the 15 already known and determined species are widely distributed. Another 4 species occur in the southern part of the southern hemisphere: South America, Australia, New Zealand and in the Subantarctic and Antarctic region. 6 species are known only from the cold water coasts of South America (Humboldt and Falkland Currents). Exogone obtusa tasmanica, Lumbrineris patagonica and Spiochaetopterus costarum are recorded from Chile for the first time.
\end{abstract}

\section{EINLEITUNG}

Die vorliegende Arbeit befaßt sich mit einer kleinen Anzahl von Polychaeten, die von K. Reise im Januar 1989 in der Gezeitenbucht "Bahia Quillaipe" südlich von Puerto Montt (Süd-Chile, s. Abb. 1) gesammelt wurde. Eine Gesamtübersicht über die Makrofauna dieser Bucht und ihre ökologischen Zusammenhänge wird von $\mathrm{K}$. Reise und $\mathrm{E}$. Clasing in Kürze folgen.

\section{BESCHREIBUNG DER ARTEN}

Familie Phyllodocidae Williams, 1852

Unterfamilie Eteoninae Bergström, 1914

Gattung Eteone Savigny, 1820

Eteone sculpta Ehlers, 1897

1897 Eteone sculpta Ehlers: 33, Taf. 1, Fig. 26-33.

1900 Eteone crassifolia Ehlers: 211.

1962a Eteone rubella, - Hartmann-Schröder: 72, Fig. 31-33.

\footnotetext{
- Mitglied der Taxonomischen Arbeitsgruppe an der Biologischen Anstalt Helgoland

- Korrespondenzanschrift 


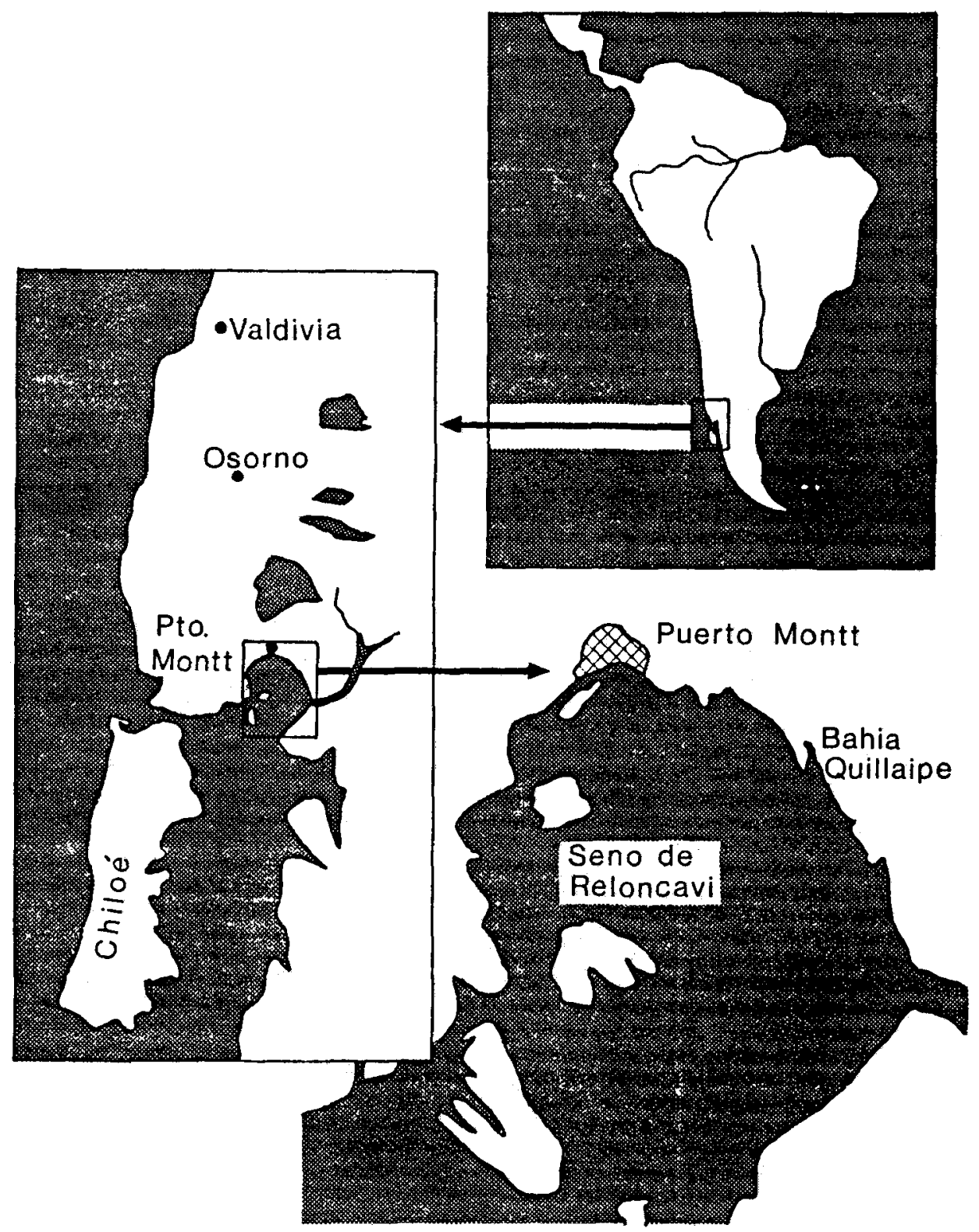

Abb. 1. Karte mit dem Untersuchungsgebiet

1964 Eteone sculpta, - Hartman: 53, Taf. 15, Fig. 9-10.

1974 Eteone sculpta, - Orensanz: 20.

Ein hinten unvollständiges Tier ist $24 \mathrm{~mm}$ lang. Färbung kräftig rötlich-braun. Vorderende wie bei Hartmann-Schröder (1962a). Mittlere Dorsalcirren nur wenig länger als breit, zum Teil leicht asymmetrisch. Rüssel vorgestülpt, mit querliegenden Wülsten. 
Fund ort: Bahia Quillaipe: Schlick, Eulitoral; 05. 01. 1989: 1 Exemplar.

V e r b r e it u n g : Mittel- und Süd-Chile, Süd-Argentinien, Falkland-Inseln, Subantarktische Inseln, Antarktis.

Ökologie: Substrat: alle Sedimente von Kies bis Schlick, Steine, Rhizoide von Macrocystis; Tiefe: Eulitoral bis $385 \mathrm{~m}$.

Familie Syllidae Grube, 1850

Unterfamilie Exogoninae Rioja, 1925

Gattung Exogone Oersted, 1845

Exogone obtusa tasmanica Hartmann-Schröder, 1989

1989 Exogone obtusa tasmanica Hartmann-Schröder: 31, Abb. 38-43.

Größtes Tier mit 41 Borstensegmenten 3,2 mm lang. Färbung gelblich. Prostomium mit Palpen, Antennen und Augen wie beim Holotypus von Tasmanien. Allerdings Hauptaugen nur bei 2 Vorderenden so groß wie dort; bei den $\$$ und neutralen Tieren nur halb so groß. Möglicherweise handelt es sich bei den Vorderenden mit großen Augen um o $\delta$. Papille zwischen Tentakelcirren und Augen hier nicht zu erkennen, da die Tiere etwas angetrocknet und geschrumpft waren. Dorsalcirren auch andeutungsweise flaschenförmig. Form der zusammengesetzten und der einfachen Borsten wie beim Holotypus. Zahl der Borsten in vorderen Parapodien 12-14. Pharynx über 31/2-41/2 Segmente; Zahn unmittelbar hinter dem Eingang. Magen über 2 Segmente, mit 21-23 Muskelzellreihen; dahinter kleiner "Nachmagen". + mit unreifen Eiern ab 12. Borstensegment.

Fund ort: Bahia Quillaipe: Sandwatt; 15. 01. 89: 8 Exemplare.

Verbre itung: Tasmanien, Süd-Chile. Die Art wurde erstmals in Chile gefunden. Ökologi e : Substrat: reiner bis kiesiger Sand; Tiefe: Eulitoral.

Familie Nereididae Johnston, 1865

Unterfamilie Nereidinae Johnston, 1865

Gattung Perinereis Kinberg, 1866

Perinereis nuntia vallata (Grube, 1857)

1857 Nereis vallata Grube: 159.

1901a Nereis vallata, - Ehlers: 110.

1901b Nereis vallata, - Ehlers: 260.

1961 Perinereis vallata, - Wesenberg-Lund: 79, Fig. 29.

1962b Perinereis vallata, - Hartmann-Schröder: 412.

1962c Perinereis vallata, - Hartmann-Schröder: 108.

1962 Perinereis vallata, - Hartmann-Schröder \& Hartman: 53.

1965 Perinereis vallata, - Hartmann-Schröder: 148, 298.

1973 Perinereis vallata, - Rozbaczylo \& Castilla: 225, Fig. 4a-f.

1974 Perinereis nuntia vallata, - Orensanz: 33.

Größtes Exemplar $64 \mathrm{~mm}$ lang. Färbung grünlich, besonders im Vorderkörper. Körper angeschwollen, eventuell durch Spermatozyten? Jedoch noch keine subepitoke Lappenbildung und noch keine vergrößerten Augen. Paragnathen: V $=1, V I=13$, $\mathrm{VII}+\mathrm{VIII}=\mathrm{ca} .50$ in 2 unregelmäßigen Reihen. 
Fu n d ort: Bahia Quillaipe: Schlick, oberes Eulitoral; 05. 01. 89: 8 Exemplare.

Verbreitung: Mittelmeer, Rotes Meer, Indopazifik, Süd- und Südwest-Afrika, Australien, Neuseeland, Mittel- bis Süd-Chile, Subantarktische Inseln.

Ökologie: alle Sedimente, wobei Schlick-Sand-Gemische bevorzugt werden, Sediment zwischen Algen, Seegras und Muscheln; Tiefe: Eulitoral bis oberstes Sublitoral.

Gattung Platynereis Kinberg, 1866

Platynereis australis (Schmarda, 1861)

1861 Heteronereis australis Schmarda: 101, Fig. a-c, k, Taf. 31, Fig. 242.

1961 Platynereis magalhaensis, - Wesenberg-Lund: 85, Fig. 33-34.

1962b Platynereis australis, - Hartmann-Schröder: 427, Fig. 44-47.

1964 Platynereis magalhaensis, - Hartman: 102, Taf. 31, Fig. 8-9.

1965 Platynereis australis, Hartmann-Schröder: 148.

1967 Platynereis magalhaensis, - Hartman: 69.

Kleines Tier mit 45 Borstensegmenten, $14 \mathrm{~mm}$ lang. Lebendfärbung grün. Parapodien und Borsten typisch.

Fund ort: Bahia Quillaipe: Sandwatt; 10. 01. 89: 1 Exemplar.

Verbreitung: Mittel- bis Süd-Chile, Ostpatagonien, Südafrika, südliches Australien, Neuseeland, Subantarktische Inseln, Antarktis.

Ö kologie: Substrat: Algen, Felsbewuchs, Rhizoide von Macrocytis, unter Steinen, seltener auf sandigen oder schlickigen Sedimenten; Tiefe: Eulitoral bis $600 \mathrm{~m}$.

Familie Nephtyidae Grube, 1850

Gattung Aglaophamus Kinberg, 1866

Aglaophamus peruana (Hartman, 1940)

(Abb. 2)

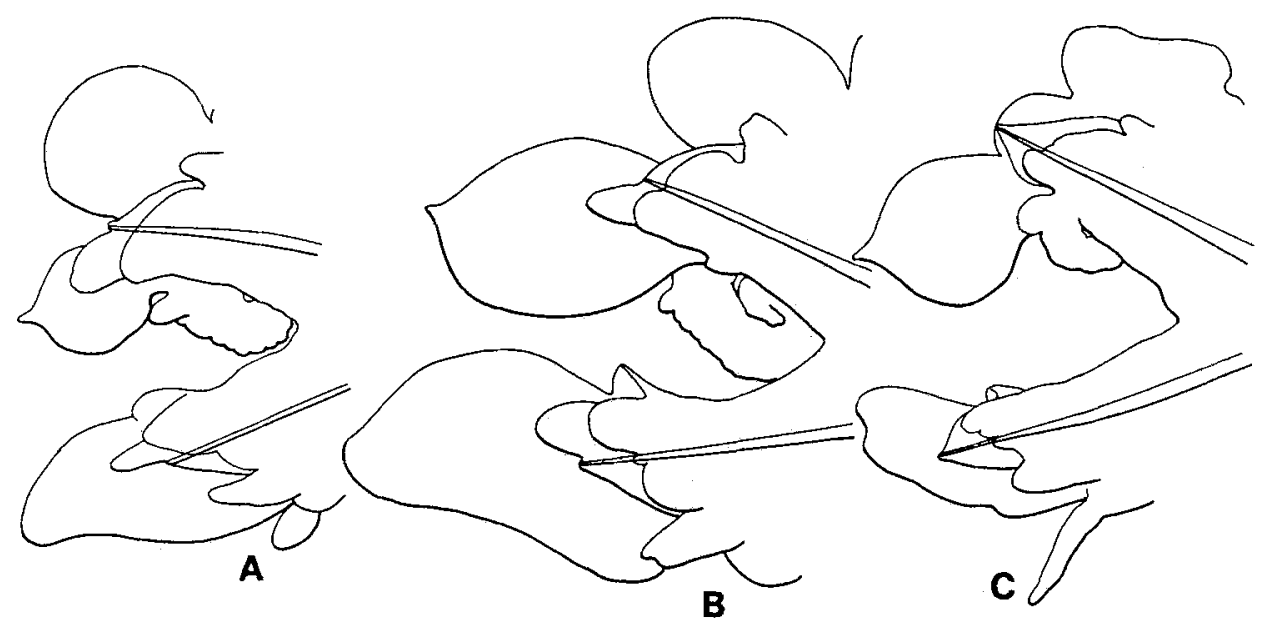

Abb. 2. Aglaophamus peruana. A: 12. Parapod von vorn. B: 28. Parapod von vorn. C: 55. Parapod von vorn 
1940 Nephthys macroura peruana Hartman: 236, Taf. 39, Fig. 89-90, Taf. 40, Fig. 96-97. ?1961 Aglaophamus macroura (non Schmarda), - Wesenberg-Lund: 95, Fig. 40-43.

1965 Aglaophamus macroura (non Schmarda), - Hartmann-Schröder: 134, Abb. 102-103.

1967 Aglaophamus peruana, - Hartman: 78.

1974 Nephtys (A.) peruana, - Rozbaczylo \& Castilla: 189.

Ein Exemplar mit 78 Borstensegmenten $27 \mathrm{~mm}$ lang. Rücken mit rötlich-, weiter hinten gelblich-bräunlichem Pigment. Prostomium mit länglichem Fortsatz am Hinterrand. Kiemen ab 3. Parapod, zunächst winzig; fehlen nur in wenigen hinteren Segmenten. Aciculäre Lappen der Noto- und Neuropodien im Vorderkörper mit je einem ovalen bis fingerförmigen Lappen unterhalb der Acicula (Notopod) bzw. oberhalb der Acicula (Neuropod) (Abb. 2A, B); im Hinterkörper aciculäre Lappen dreieckig (Abb. 2C). Präsetale Lappen kürzer als die aciculären, zwei- bis mehrlappig (Abb. 2A-C). Postsetale Lappen der Noto- und Neuropodien am weitesten vorragend: die dorsalen breit gerundet, im Hinterkörper zweilappig; die ventralen mehr oder weniger zungenförmig (Abb. 2A-C). Notopodialer Cirrus sehr groß, besonders in mittleren Segmenten, zugespitzt (Abb. 2A-C). Rüssel wie abgebildet bei Wesenberg-Lund (1961, Fig. 40).

Fun d ort: Bahia Quillaipe: Sand; Januar 1989: 1 Exemplar.

Verbreitung: Peru bis Süd-Chile.

Ökologie: Substrat: Grob- bis Feinsand mit oder ohne Schlickbeimengung; Tiefe: $6-300 \mathrm{~m}$.

Taxonomische Bemerkung: Die vorliegende Art ist wiederholt mit der nahe verwandten $A$. macroura (Schmarda) verwechselt bzw. identifiziert worden. Hartman (1967) unterscheidet $A$. peruana von der neuseeländischen $A$. macroura hauptsächlich durch die Fortsätze der aciculären Lappen (s. oben), die nach dem etwa 39. Parapod allmählich verschwinden. Diese Fortsätze sind auch bei der A. macroura von HartmannSchröder (1965) vorhanden. Die Form der Parapodien der 'A. macroura von WesenbergLund (1961) ist auch sehr ähnlich; es sind jedoch keine Einzelheiten zu erkennen, da die Parapodien in der Hinteransicht gezeichnet wurden. Da nirgends Parapodien-Abbildungen mit allen Details vorliegen, werden hier genaue Zeichnungen vorgelegt.

Gewisse Ähnlichkeit besitzt auch A. foliosus Hartman, besonders im notopodialen präsetalen Lappen, im Neuropod und in der Form des notopodialen Cirrus. Die aciculären Lappen sind jedoch anders, Kiemen beginnen erst im 4. Parapod, und die Rüsselpapillen spalten sich an der Basis nicht in 2 Reihen winziger Papillen auf, wie es für $A$. peruana typisch ist.

Aglaophamus virginis (Kinberg, 1866) sensu Hartman, 1967

1866 Nephthys virginis Kinberg: 229.

1962a Aglaophamus virginis, - Hartmann-Schröder: 54.

1964 Aglaophamus virginis, - Hartman: 103, Taf. 32, Fig. 2.

1967 Aglaophamus virginis, - Hartman: 79, Taf. 26.

1974 Nephtys (A.) virginis, - Rozbaczylo \& Castilla: 193.

Ein Tier mit zahlreichen Segmenten $115 \mathrm{~mm}$ lang. Nur ganz schwach gelblichbräunliches Pigment auf dem Rücken. Prostomium genau so wie in Fig. 26A von Hartman (1967). Im Hinterkörper Segmente dorsolateral oberhalb der Parapodien am Hinterrand etwas häutig verlängert und der notopodiale postsetale Lappen der Parapodien besonders groß, das jeweils folgende Parapod überlappend. Kiemen ab 2. Parapod, nur in den 
letzten 10-12 Segmenten fehlend. Parapodien wie in Fig. 26B von Hartman (1967): besonders charakteristisch der notopodiale postsetale Lappen, der im oberen Teil groß, gerundet und darunter in 2 finger- bis fadenförmige Lappen unterteilt ist; ab 6. Parapod mit 1 fadenförmigem Lappen beginnend; danach der obere meist etwas kürzer als der untere; ab etwa 40. Parapod oberer finger- oder fadenförmiger Lappen schnell kleiner werdend und danach nur noch der ehemals untere vorhanden, der als Lappen dreieckig und fadenförmig ausgezogen. Notopodialer Cirrus hier schlank, lanzeolat.

Fund ort: Bahia Quillaipe: Sand; Januar 1989: 1 Exemplar.

Verbreitung: Süd-Chile, Süd-Argentinien, Falkland-Inseln, Neuseeland, Subantarktische Inseln, Antarktis.

Ökologi e: Substrat: wenig bekannt, Sand; Tiefe: Eulitoral bis $370 \mathrm{~m}$.

Taxonomische Bemerkung: Hartman (1967) schreibt "restricted" hinter den Artnamen, gibt aber weiter keine Begründung dafür. 1953 gibt Hartman an, daß sie das Kinbergsche Originalmaterial gesehen hat, von dem sie schreibt, daß die Kiemen ab "about" 4. Segment auftreten, erwähnt aber nicht die fingerförmigen Lappen des notopodialen postsetalen Lappens. Ihre Fig. $7 \mathrm{a}$ und $\mathrm{b}$ stammen vermutlich von ihren eigenen Tieren und stellen beide das 24. Parapod von vorn und hinten dar. Das entspricht im Neuropod auch der Fig. 26B von Hartman (1967). Im Notopod ist der notopodiale Cirrus jedoch viel größer, und die finger- oder fadenförmigen Lappen der postsetalen Lappen sind nicht vorhanden. Ohne das Kinbergsche Material nachzuuntersuchen, ist es nicht sicher, ob Hartman (1967) die wahre $A$. virginis beschreibt und ob Hartman (1953) die Einzelheiten der Parapodiallappen nicht genau erkannt hat.

Familie Glyceridae Grube, 1850

Gattung Hemipodus Quatrefages, 1865

Hemipodus heteropapillatus Hartmann-Schröder, 1962

1962a Hemipodus heteropapillatus Hartmann-Schröder: 111, Fig. 109-112.

1965 Hemipodus heteropapillatus, - Hartmann-Schröder: 149.

Größtes Tier etwa $52 \mathrm{~mm}$ lang (etwas geknäuelt). Vordere Segmente kaum, mittlere etwas und hintere stärker mit sehr feinen gelblich-grünlich-bräunlichen Pigmentkörnchen bedeckt. Beim größeren Exemplar Segmente ab 19., bei den kleineren ab 15. Borstensegment dreiringelig; im mittleren Körper Segmente zwei- oder undeutlich dreiringelig, weiter hinten wieder deutlich dreiringelig. Parapodien wie beim Holotypus, auch präsetale Lappen und Ventralcirren der hinteren Parapodien verlängert und spitz dreieckig. Proboscideale Organe wie beim Holotypus.

Fund ort: Bahia Quillaipe: Sand, unteres Eulitoral; 05. 01. 89: 3 Exemplare.

Verbreitung: Nord- bis Süd-Chile.

Ök ologi e: Substrat: Feine bis kiesige Sande, mit oder ohne Schlickbeimengungen; Tiefe: Eulitoral bis $58 \mathrm{~m}$.

Familie Lumbrineridae Malmgren, 1867

Gattung Lumbrineris Blainville, 1828

Lumbrineris patagonica Hartmann-Schröder, 1962 
1962a Lumbrineris patagonica Hartmann-Schröder: 119, Abb. 124-127.

1974 Lumbrineris patagonica, - Orensanz: 38.

Das einzige vollständige Exemplar ist etwa $60 \mathrm{~mm}$ lang. Ein dickeres mittleres Bruchstück mißt $70 \mathrm{~mm}$. Färbung fleischfarben, irisierend. Form der Parapodien und Borsten wie beim Holotypus. Einfache Hakenborsten ab 20. Parapod; ab 34. Parapod nur noch Hakenborsten vorhanden. Maxillen: II $=4+4$, III $=2+2$, IV $=1+1$.

Fund ort: Bahia Quillaipe: Sand, unteres Eulitoral; 09. 01. 89: 5 Exemplare.

Verbreitung: Patagonien (Süd-Argentinien), Süd-Chile. Die Art ist erstmals in Chile gefunden.

Ö kologi e : Substrat: Sand, Lehm, auch im Sediment zwischen Algen und Steinen; Tiefe: Eulitoral.

Familie Orbiniidae Hartman, 1942

Unterfamilie Orbiniinae Hartman, 1942

Gattung Scoloplos Blainville, 1828

Scoloplos (Leodamas) tribulosus (Ehlers, 1897)

1897 Aricia tribulosa Ehlers: 91, Taf. 6, Fig. 141-147.

1962a Scoloplos (L.) tribulosus, Hartmann-Schröder: 129, Abb. 151-153.

1965 Scoloplos (L.) tribulosus, Hartmann-Schröder: 192.

1966 Scoloplos (L.) tribulosus, Hartman: 13, Taf. 3, Fig. 1-2.

1969 Scoloplos (L.) tribulosus, Ringuelet: 210.

1974 Scoloplos (L.) tribulosus, Orensanz: 50.

Größtes Vorderende mit 21 Thoraxsegmenten mit Borsten und 50 Abdominalsegmenten $36 \mathrm{~mm}$ lang. Färbung gelblich-weiß. Kiemen ab 5. Borstensegment. Anderes Vorderende mit 23 Borstensegmenten im Thorax und Kiemen ab 6. Borstensegment. Form der Parapodien und Gabelborsten mit denen des Holotypus übereinstimmend.

Fu n d ort: Bahia Quillaipe: Sand; 05. 01. 89: 7 Exemplare, 5 davon juvenil.

Verbre it ung: Mittel- und Süd-Chile, Süd-Argentinien.

Ökologi e: Substrat: Sand, Mischböden aus Sand und Schlick; Tiefe: Eulitoral bis $58 \mathrm{~m}$.

Familie Spionidae Grube, 1850

Gattung Polydora Bosc, 1802

Polydora (Boccardia) polybranchia Haswell, 1885

1885 Polydora (Leucodore) polybranchia Haswell: 275.

1962a Boccardia polybranchia, - Hartmann-Schröder: 134, Abb. 165-166.

1965 Boccardia polybranchia, - Hartmann-Schröder: 202.

1966 Boccardia polybranchia, - Hartman: 15, Taf. 3, Fig. 11-12.

1974 Boccardia polybranchia, - Orensanz: 42.

1983 Boccardia polybranchia, - Blake: 248.

Größtes vollständiges Tier etwa $7 \mathrm{~mm}$ lang (aufgerollt). Etwas dunkelbraunes Pigment auf Prostomium und vorderen Segmenten. Prostomium vorn eingeschnitten. 1. Notopod ohne Borsten. 5. Segment mit jederseits 2 glatten falcaten und 3 subdistal verbreiterten, abgestutzten und behaarten Spezialborsten. Kiemen fehlen im Hinterkörper. Pygid mit 4 runden Lappen. Bruchstücke von braunen Schlickröhren vorliegend. 
Fu n d ort: Bahia Quillaipe: Schlick; 07. 01. 89: 5 Exemplare.

Verbreitung: weit verbreitet, besonders auf der Südhalbkugel: von den Tropen bis zu den Subantarktischen Inseln; Nord- bis Südchile.

Ökologie: Substrat: alle Sedimente, Phytal, Felsbewuchs, unter Steinen, zwischen Muscheln und Sabellariiden-Röhren, in Rockpools; Tiefe: Eulitoral bis $58 \mathrm{~m}$. Die Art ist euryhalin und kommt auch im Süßwasser vor.

\section{Gattung Spiophanes Grube, 1860}

Spiophanes bombyx (Claparède, 1870)

1870 Spio bombyx Claparède: 485 , Taf. 12, Fig. 2.

1966 Spiophanes bombyx, - Hartman: 22, Taf. 5, Fig. 14-16.

1974 Spiophanes bombyx, - Carrasco: 197, Fig. 37-41.

1974 Spiophanes bombyx, - Orensanz: 43.

1975 Spiophanes bombyx, - Carrasco: 7.

1976 Spiophanes bombyx, - Carrasco: 48, Fig. 19, 20, 22J-L.

1983 Spiophanes bombyx, - Blake: 230.

Größtes Vorderende $6 \mathrm{~mm}$ lang. Prostomium mit langen frontalen "Hörnern". Keine Occipitalantenne. Hakenborsten und Stachelförmige Borste ab 15. Neuropod. Hakenborsten mit kleinen Scheitelzähnen; Kapuze hauptsächlich unterhalb des Hauptzahns zu erkennen. Genitaltaschen ab 5. Borstensegment.

Fund or t: Bahia Quillaipe: Sand, unteres Eulitoral; 21. 01. 89: 2 Exemplare.

Verbreitung: Außer Arktis und Antarktis weltweit; Mittel-bis Süd-Chile.

Ökologie: Substrat: Grobsand bis Schlick, Mischböden, Sediment zwischen Rhizoiden von Laminarien und Austern; Tiefe: Eulitoral bis $200 \mathrm{~m}$.

\section{Gattung Scolelepis Blainville, 1828}

Scolelepis (Scolelepis) brevibranchia sp. $\mathrm{n}$.

Der Hol oty p u s ist ein Vorderende mit 55 Borstensegmenten; Länge etwa $11 \mathrm{~mm}$ (aufgerollt). Etwas braunes Pigment auf der Mitte des Prostomiums vor den Augen. Lebend war das Tier intensiv orange gefärbt, mit dunklem Darminhalt. Prostomium zugespitzt, nach hinten in eine kielartig erhabene Karunkel auslaufend, die bis zur Mitte des 2. Borstensegments reicht. Auf dem höchsten Punkt der kielartigen Karunkel eine Occipitalantenne vorhanden (Abb. 3A). Vor der Occipitalantenne 4 kleine Augen in einer Reihe (sowohl Augen als auch Pigment während der Tage der Untersuchung verschwunden). 1. Notopod nur aus winzigem Lappen bestehend, ohne Borsten. Kiemen ab 2. Parapod, am ganzen Körper sehr kurz und bis auf kleinen distalen Zipfel mit dem breit gerundeten, teilweise leicht gewellten postsetalen Lappen der Notopodien verwachsen (Abb. 3B-E). Unterhalb des notopodialen postsetalen Lappens ein zunächst kleiner, an der Basis eingeschnürter, breit gerundeter interramaler Lappen, der nach hinten zu etwas kürzer, aber höher und an der Basis nicht mehr eingeschnürt ist (Abb. 3B-E). Neuropodialer postsetaler Lappen flach, breit gerundet, in mittleren Segmenten zweilappig (Abb. 3B-E). Notopodiale Borsten in 2 Reihen: in vorderen Segmenten wenig unterschiedlich, gesäumt und teilweise gepunktet. Im 20. Notopod in der vorderen Reihe 6 kürzere, dünnere und in der hinteren Reihe 7 etwas längere, dickere schmal gesäumte Borsten. In hinteren vorhandenen Segmenten auch die längeren Borsten nicht mehr 


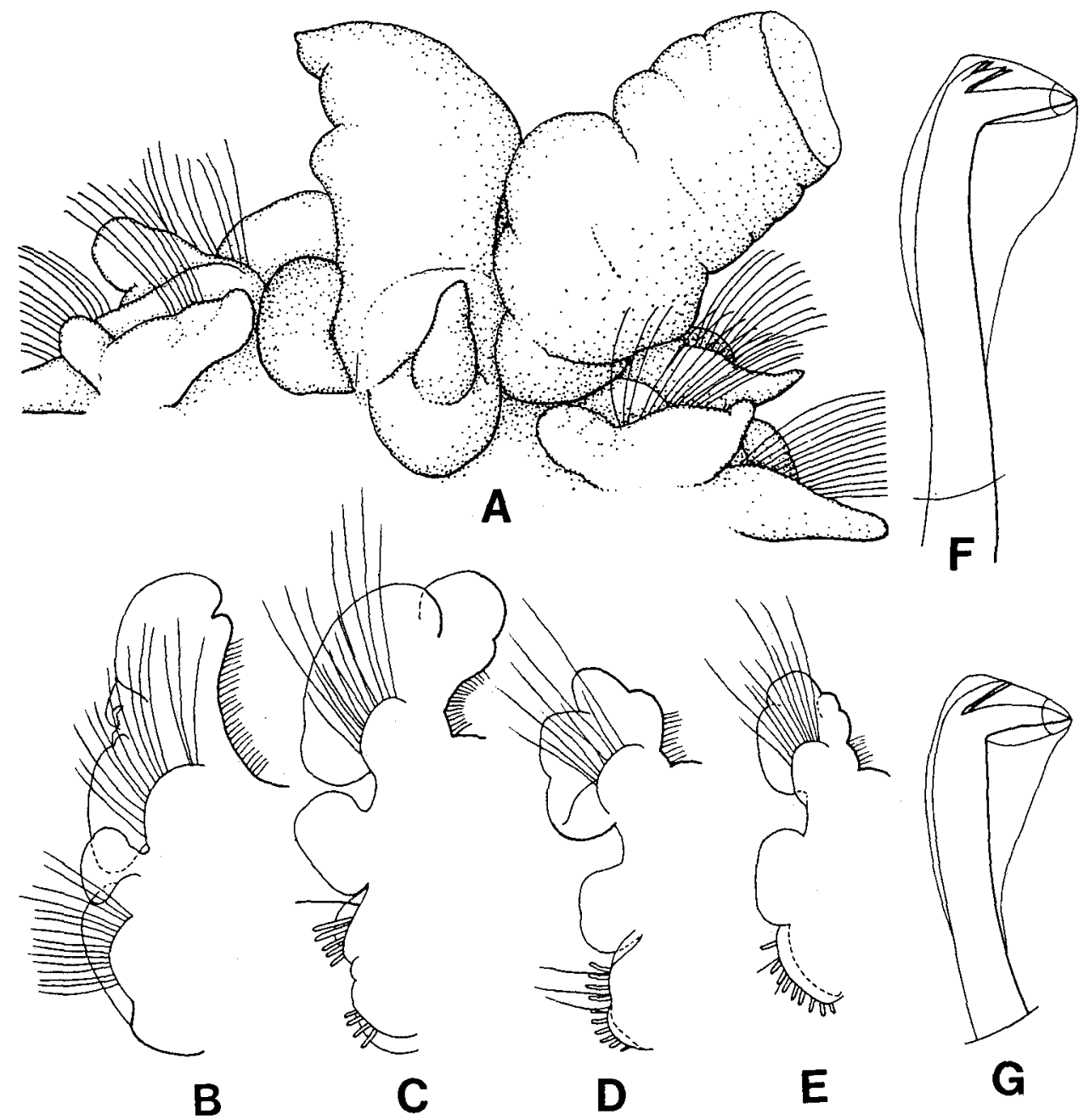

Abb. 3. Scolelepis (S.) brevibranchia sp. n. A: Vorderende von dorsal (linker Tentakel abgebrochen). B: 7. Parapod von vorn. C: 20. Parapod von vorn. D: 30. Parapod von vorn. E: 55. Parapod von vorn. F: mittlere Hakenborste aus hinterem Neuropod. G: Untere Hakenborste aus hinterem Neuropod

gesäumt. Neuropodiale Borsten in vorderen Segmenten in mehreren Reihen angeordnet, etwas breiter als die dorsalen, gesäumt und zum Teil gepunktet. Ab 15. Neuropod treten 2 Hakenborsten auf. Im 20. Neuropod bereits 9 Hakenborsten vorhanden: die oberen und unteren mit 1 Paar Scheitelzähnen nebeneinander, die mittleren mit 2 Paar (Abb. 3F-G); dazu eine dünne ungesäumte Borste oberhalb der Hakenborsten. Im 30. Neuropod 10 Hakenborsten, von denen mindestens 3 untere mit 1 Paar Scheitelzähnen; dazu 3 dünne Borsten im oberen Teil des Neuropods. Im letzten vorhandenen Neuropod (55.) 9 Hakenborsten, von denen die oberste und 2 untere 1 Paar Scheitelzähne, die mittleren 
2 Paar aufweisen; dazu 2 dünne Borsten. Nicht nachweisbar, ob in hinteren, hier fehlenden Segmenten notopodiale Hakenborsten auftreten.

L o cus typicus: Bahia Quillaipe: Schlickwatt, oberes Eulitoral; 13. 01. 89: Holotypus (ZMH:P-20 219).

Tax on om is che Bemerkung: Gewisse Ähnlichkeit besteht mit $S$. (S.) quinquedentata Hartmann-Schröder im Vorhandensein einer Occipitalantenne und einer interramalen Lamelle; auch die Form der Hakenborsten stimmt überein; aber die interramale Lamelle ist nicht deutlich vom neuropodialen postsetalen Lappen getrennt, und im 23. Borstensegment sind Kieme und dorsaler postsetaler Lappen schon deutlich separat. Auch bei S. (S.) sp. von Hartmann-Schröder (1980) ist eine interramale Lamelle vorhanden. Dort sind jedoch Kieme und dorsaler postsetaler Lappen nur an der Basis miteinander verwachsen, und die Hakenborsten sind einspitzig bis undeutlich zweispitzig.

Hingewiesen werden muß noch auf die Beobachtung von Reise, daß die Kiemen am lebenden Tier teilweise etwas länger erscheinen als im fixierten Zustand. Da dem Taxonom jedoch in den allermeisten Fällen fixiertes Material vorliegt, halte ich den Namen brevibranchia für gerechtfertigt (s. Abb. 3B-E).

\section{Scolelepis (Scolelepis) crenulata sp. $\mathrm{n}$.}

Holotypus: $q$ mit Eiern, mit 156 Borstensegmenten $118 \mathrm{~mm}$ lang; größte Breite 4,5 mm. Bis auf etwas bräunliches Pigment lateral am vorgestülpten Rüssel fixierte Tiere farblos. Lebendfärbung nach Reise: Vorderkörper rosa, Kiemen rot, Hinterkörper gelblich, mit dunkelgrüner Seitenzeichnung zwischen den Parapodien. Prostomium zugespitzt, zusammen mit der Karunkel einen schlanken Rhombus darstellend. Karunkel erhöht, tropfenförmig, völlig mit dem Rücken verwachsen, bei den beiden Paratypen die

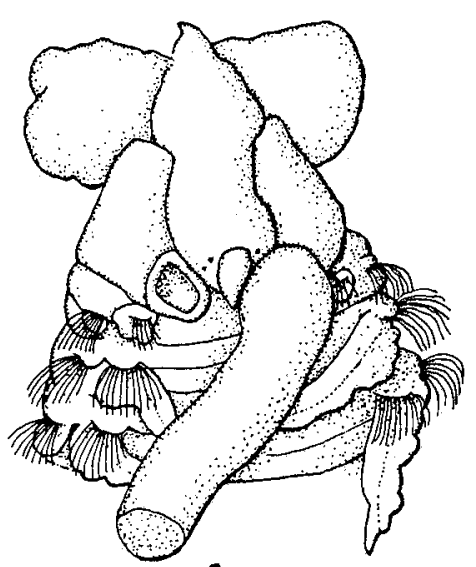

A

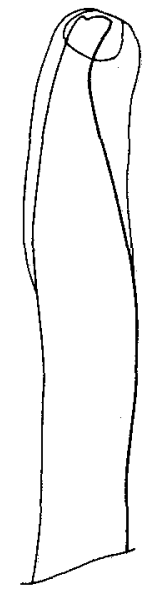

C

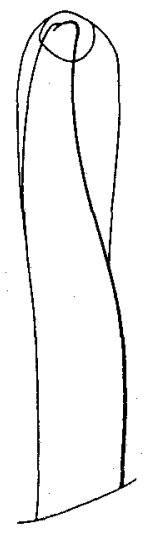

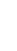

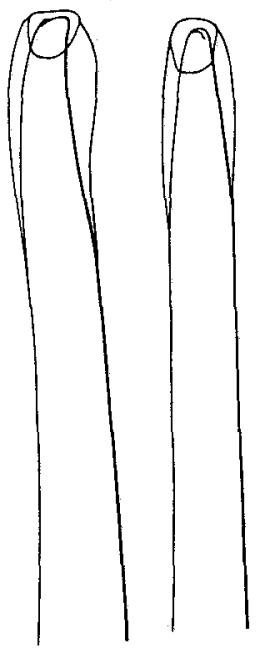

B

Abb. 4. Scolelepis (S.) crenulata sp. n. A: Vorderende eines Paratypus (linker Tentakel abgebrochen). B: Hakenborsten aus hinterem Notopod. C: Hakenborsten aus hinterem Neuropod 

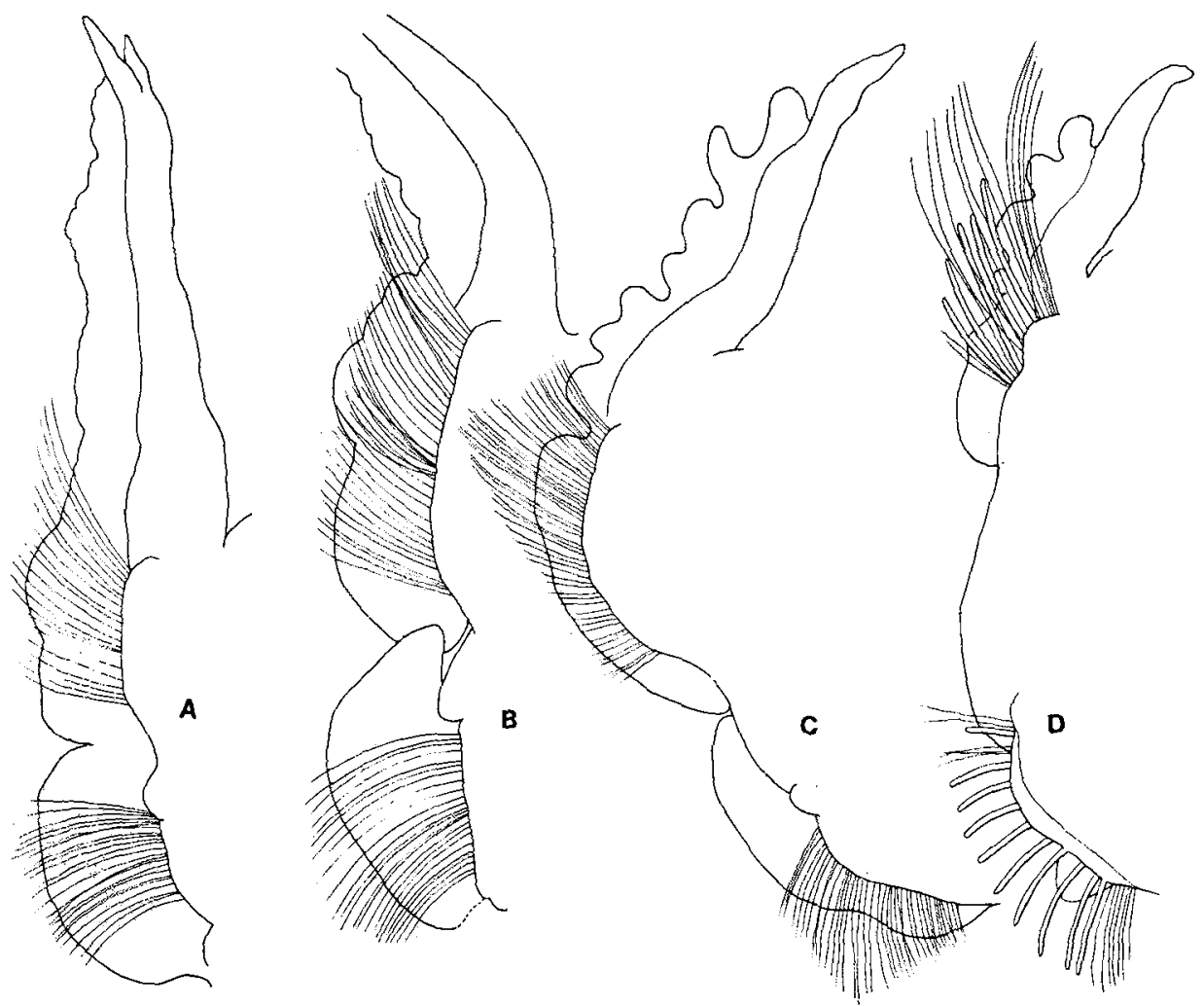

Abb.5. Scolelepis (S.) crenulata sp. n. A: 7. Parapod von vorn. B: 20. Parapod von vorn. C: 30. Parapod von vorn. D: 112. Parapod von vorn

hintere Spitze etwas frei; bis 1. Borstensegment nach hinten reichend (Abb. 4A). 2 kleine Augen auf der Höhe des Karunkelvorderrandes und 2 winzige etwas dahinter, weiter außen und tiefer unter der Haut liegend, so daß sie leicht zu übersehen sind. 1. Notopod mit kleinem gewellten postsetalen Lappen und Borsten. Kiemen ab 2. Parapod, bis auf die Spitzen mit dem stark gewellten postsetalen Lappen des Notopods verschmolzen. Kiemen und dorsale postsetale Lappen am ganzen Körper völlig miteinander verwachsen $_{i}$ nur die Kieme etwas weiter hinausragend (Abb. 5A-D). Notopodialer postsetaler Lappen überall stark gewellt, sich unten nur in vorderen mittleren Segmenten mit dem neuropodialen postsetalen Lappen etwas überlappend (Abb. 5B). Ventraler postsetaler Lappen flach, nach dem 20. Parapod schwach zweilappig, ab etwa 35. Parapod mit unterem abgerundet dreieckigen Lappen, der im Hinterkörper als einziger neuropodialer postsetaler Lappen vor dem präsetalen vorragt (Abb. 5A-D). Im Vorderkörper dorsale und ventrale Borsten sehr zahlreich, anscheinend in 2 Reihen angeordnet; sie sind gelblich, fein gepunktet und gesäumt. Weiter hinten werden die Borsten etwas dünner, bleiben gesäumt, weisen aber keine Punktierung mehr auf. Ventrale Hakenborsten treten ab etwa 34., dorsale ab etwa 50. Parapod auf. Dorsal finden sich maximal 6, ventral bis zu 12 Hakenborsten neben den Kapillarborsten. Hakenborsten bräunlich, stark 
abgenutzt, fast wie einspitzig aussehend, jedoch Rest eines Scheitelzahns zu erkennen, und zwar sowohl bei den dorsalen als auch bei den ventralen Hakenborsten (Abb. 4B-C). In den hinteren Neuropodien wenige Borsten neben den oberen Hakenborsten und ein unteres Bündel von 6-7 Borsten unterhalb der Hakenborsten. Diese unteren Borsten etwas kürzer, etwas dicker und etwas breiter gesäumt als die übrigen Borsten dieser Körperregion, außerdem sind sie sehr fein gepunktet. Hutchings \& Turvey (1984) bezeichnen diese Borsten bei ihrer $S$. (S.) bifida als "sabre setae" (Stachelförmige Borste). Es sei dahingestellt, sie mit den meist stärker gebogenen und breiteren Stachelförmigen Borsten anderer Spioniden zu vergleichen. Pygid ein ovaler bis abgerundet dreieckiger Lappen; Anus dorso-terminal.

L o cus ty picus: Bahia Quillaipe: Sandwatt, unteres Eulitoral; 22. 01. 86: Holotypus (ZMH:P-20220) und 2 Paratypen (ZMH:P-20221).

Taxonomische Bemerkung: Die größten Ähnlichkeiten mit der oben beschriebenen Art weisen S. antipoda (Augener) und S. bifida Hutchings \& Turvey auf. Bei beiden Arten sind Kiemen und dorsale postsetale Lappen weitgehend übereinstimmend. Im Gegensatz zur neu beschriebenen Art sind die ventralen Borsten und Hakenborsten der mittleren und hinteren Segmente auf den Bereich des unteren abgerundet dreieckigen neuropodialen postsetalen Lappens beschränkt, während die Borsten bei $S$. crenulata viel weiter hoch reichen (Abb. 5D). S. antipoda und $S$. bifida erscheinen mir untereinander sehr ähnlich, wenn nicht identisch; doch Hutchings \& Turvey (1984), die das Typenmaterial von $S$. antipoda nachuntersucht haben, sehen Unterschiede in dem etwas gerundeteren Prostomium von $S$. antipoda - was jedoch mit dem Fixierungszustand zusammenhängen könnte - und in den Kiemen: "The anterior branchiae of $S$. antipoda are cylindrical with a small dorsal terminal lamella(e) whereas in $S$. bifida n. sp. the branchiae are simple and cylindrical" (S. 6). - Ein Unterschied, der mir nicht klar wird. Die Abbildung des Parapods aus dem vorderen Körperdrittel bei Augener (1926, Fig. 2c) und die des 40. Parapods bei Hutchings \& Turvey (1984, Fig. 1d) erscheinen mir sehr ähnlich, wenn nicht identisch. Abgesehen von der möglichen Identität von $S$. antipoda und $S$. bifida, unterscheidet sich $S$. crenulata sp.n. von ihnen in folgenden Punkten: Die dorsalen postsetalen Lappen sind vom 1. Segment an stark gewellt, während sie bei $S$. bifida erst nach dem 20. Parapod gewellt sind wie anscheinend auch bei $S$. antipoda (vgl. Fig. 2a von Augener). Die dorsalen und ventralen postsetalen Lappen überlappen sich deutlich in der Region um das 20. Borstensegment; bei S. bifida erreichen sie einander nirgends. In mittleren und hinteren Neuropodien reichen die Borsten und Hakenborsten weiter dorsad, während sie bei $S$. antipoda und $S$. bifida auf den Bereich des unteren dreieckigen Teils der postsetalen Lamelle beschränkt sind. Abgesehen davon, sind wahrscheinlich auch Unterschiede in der Form der Karunkel vorhanden, welche auf der sehr kleinen Fig. 1a von Hutchings \& Turvey nicht genau zu erkennen sind.

Familie Chaetopteridae Malmgren, 1867

Gattung Spiochaetopterus M. Sars, 1853

Spiochaetopterus costarum (Claparède, 1868)

1868 Telepsavus costarum Claparède: 340, Taf. 20, Fig. 1.

1984 Spiochaetopterus costarum, - Gilbert: 8, Fig. 11-3, 4a-m. 


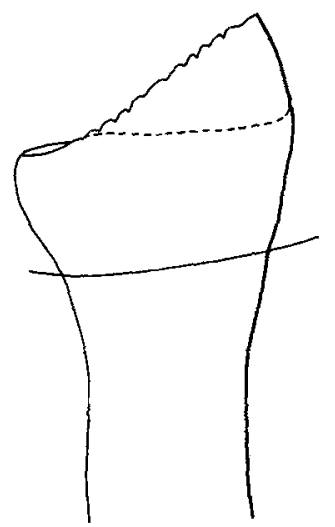

A

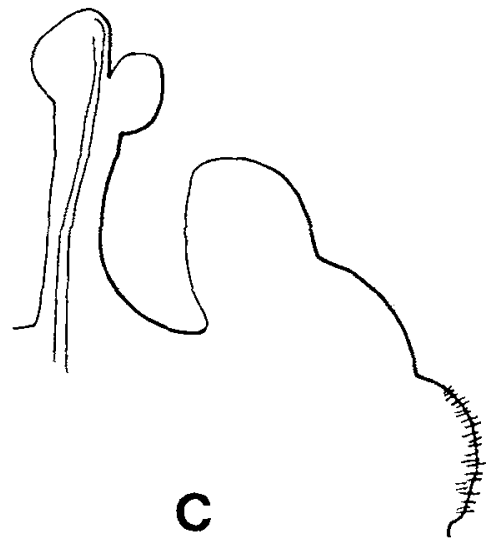

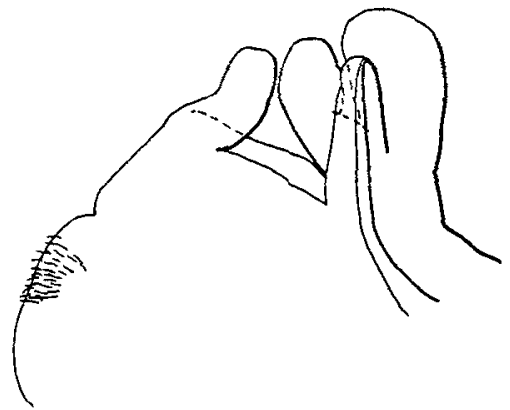

B

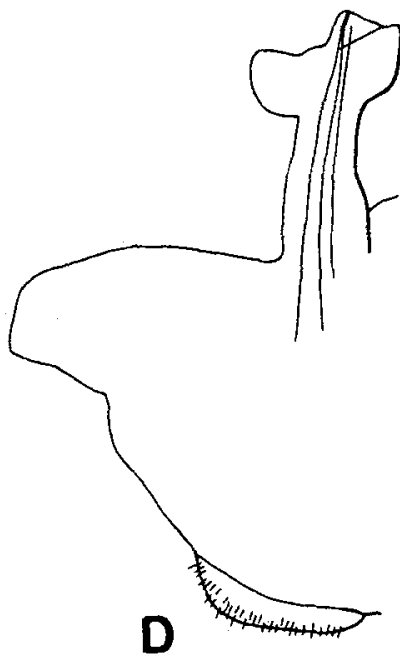

Abb.6. Spiochaetopterus costarum. A: Haken aus 4. Parapod. B: 3. Parapod aus mittlerem Körperabschnitt. C: mittleres Parapod aus mittlerem Körperabschnitt. D: hinteres vorhandenes Parapod aus mittlerem Körperabschnitt

Ein unvollständiges Tier mit 9 Borstensegmenten im Vorderkörper und 29 Segmenten des mittleren Körperabschnitts vorliegend; Länge etwa $17 \mathrm{~mm}$ (geknäuelt). Färbung im fixierten Zustand gelblich-weiß, mit sehr feinen dunkelbraunen Pigmentkömchen auf dem Peristomium, sehr schwach auf den Tentakeln und als dorsolateraler Fleck auf dem Thorax dorsal und ventral auf den vorderen 6 Segmenten; ventral dahinter ein großes dunkelbraunes, drüsiges(?), etwa halbmondförmiges Feld, das von der Mitte des 6 . bis zur Mitte des 7. Segments reicht; dahinter ein dickes weißes Polster, das sich bis zum Hinterrand des 9. Segments erstreckt. Nach Reise ist die Pigmentierung des lebenden Tieres braun-violett. Prostomium ein ganz flaches, wenig abgehobenes, nach vorn gerundetes Polster mit 2 länglichen Pigmentflecken (Augen?). Peristomium kragenartig. 4. Segment jederseits mit einem dicken bräunlichen, distal schräg abgestutzten Haken, dessen distale Kante fein gekerbt (Abb. 6A); daneben einige lanzettförmige Borsten, die distal etwas aufgesplittert. Segmente des mittleren Körperabschnitts nach hinten zu 
langsam kürzer werdend. Noto- und Neuropodien mit- und untereinander durch häutige Lamelle verbunden. Notopodien zweilappig, bewimpert, mit 2-3 eingebetteten Kapillarborsten (Abb. 6B-D). Neuropodien mit dorșad gerichtetem fingerförmigen bis dreieckigen bewimperten Lappen und flachem ventrolateralen Hakenpolster (Abb. 6B-D). Röhre hornig, transparent, teilweise bräunlich, geringelt, $114 \mathrm{~mm}$ lang.

Fun d or t: Bahia Quillaipe: Sand, unteres Eulitoral; 05. 01. 89: 1 Exemplar.

Verbreitung: Außer Arktis und Antarktis/Subantarktis weltweit. Die Art ist ein Neufund für Chile.

Ökologie: Substrat: Sand, Schlick, Mischböden, Lehm, Korallengrund, unter Steinen; Tiefe: Eulitoral bis $378 \mathrm{~m}$.

Taxonomische Bemerkung: Das vorliegende Tier stimmt gut mit $S$. costarum überein: in der Zahl der mittleren Segmente, in der Form des Peristomiums, der Parapodien der mittleren Segmente und der Haken des 4. Segments. Die Unterart oculatus Webster ist nach Gitay (1969) fast identisch mit der Nominatform, die Unterarten monroi Gitay und pottsi (Berkeley) unterscheiden sich von der Stammart nur in der Form der Neuropodien des Hinterkörpers (hier nicht vorliegend); und die Unterart okadai Gitay hat 67 Segmente im mittleren Körperabschnitt (hier nur bis 29 vorliegend). Da hier die Zahl der mittleren Segmente nicht feststellbar ist und das Hinterende fehlt, ist das vorliegende Exemplar der Stammart zugeordnet worden.

\section{Familie Cirratulidae Carus, 1863 \\ Gattung Cirriformia Hartman, 1936}

Cirriformia quetalmahuensis Hartmann-Schröder, 1962

1962a Cirriformia quetalmahuensis Hartmann-Schröder: $14^{\prime} 4$.

1965 Cirriformia quetalmahuensis, - Hartmann-Schröder: 222.

1974 Cirriformia quetalmahuensis, - Orensanz: 46.

Größtes Tier etwa $40 \mathrm{~mm}$ lang. Färbung in Alkohol grau-gelblich, nach Reise im Leben rotbraun, Kiemen rot. Tentakelfilamente in querliegendem, schmal-dreieckigen Haufen jederseits auf dem 5. und 6. Borstensegment. Kiemen dicht über den Notopodien entspringend. Ventrale Haken ab 24. Parapod; im Hinterkörper nur noch Haken vorhanden.

Fund ort: Bahia Quillaipe: Schlicksand, 05. 01. 89: 5 Exemplare.

Verbreitung: Mittel- bis Süd-Chile.

Ökologie: Substrat: Sand mit Schlick, mit oder ohne Steine, Sand zwischen Algen; Tiefe: Eulitoral bis oberstes Sublitoral.

Familie Opheliidae Malmgren, 1867

Unterfamilie Travisiinae Hartmann-Schröder, 1971

Gattung Travisia Johnston, 1840

Travisia olens Ehlers, 1897

1897 Travisia olens Ehlers: 98, Taf. 6, Fig. 162-163.

1966 Travisia olens, - Hartman: 55, Taf. 17, Fig. 11.

1974 Travisia olens, - Orensanz: 48. 
Größtes Tier mit 26 Borstensegmenten und 3 hinteren borstenlosen Segmenten $26 \mathrm{~mm}$ lang. Größte Breite $6 \mathrm{~mm}$. Färbung blaß fleischfarben. Nach Reise einen unangenehmen, knoblauchähnlichen Geruch ausströmend. Ab 2. Borstensegment Segmente dreiringelig, hintere 5 Borstensegmente zweiringelig und die letzten 3 borstenlosen Segmente nicht sekundär geringelt. Hintere 9-10 Segmente, inklusive die 3 borstenlose Segmente, dorsolateral am Hinterrand undeutlich bis kaum sichtbar krenuliert. Lanzettbis cirrenförmige postsetale Lappen (oder Kiemen) ab 2. Parapod, an hinteren borstenlosen Segmenten fehlend. Laterale Lappen sehr klein und rundlich ab 3. Parapod; ab 9. Parapod etwas deutlicher, aber immer noch klein und gerundet; danach langsam größer werdend; ab 15. Parapod oval, ab 19. oval und noch etwas dicker; schließlich nur noch wenig dicker werdend. Anus terminal, umgeben von 8 verschieden dicken wulstartigen Papillen. Sehr gute Übereinstimmung mit T. olens von der Magellan-Straße (ZMH: V.4865).

Fundort: Bahia Quillaipe: Grobsandwatt, unteres Eulitoral; 09. 01. 89; 4 Exemplare.

Verbreitung: Süd-Chile, Süd-Argentinien, Falkland-Inseln, Subantarktische Inseln, Antarktis, West-Australien, Neuseeland.

Ökologie: Substrat: Fein- bis Grobsand; Tiefe: Eulitoral bis $1120 \mathrm{~m}$.

Familie Maldanidae Malmgren, 1867

Unterfamilie Euclymeninae Arwidsson, 1906

Gattung Euclymene Verrill, 1900

Euclymene (Euclymene) sp.

Nur Bruchstücke vorliegend. Folgende Teile mögen zusammengehören und ein komplettes Tier darstellen: 1 Vorderende mit 8 Borstensegmenten, 2 folgende Segmente (9. und 10.), 6 vordere mittlere, 5 hintere mittlere Segmente sowie 1 Hinterende mit 3 Borsten- und 1 borstenlosen präanalen Segment; zusammen 24 Borstensegmente und 1 präanales borstenloses Segment; Länge etwa $76 \mathrm{~mm}$; größte Breite 1,9 mm. Färbung in Alkohol rosa-fleischfarben; nach Reise lebendes Tier leuchtend rot. Kopfplatte mit hohen, glatten Säumen, die lateral und hinten eingeschnitten sind (Abb. 7 A). Nuchalgruben lang, parallel, nur vorn schwach divergierend, fast über $3 / 4$ der Kopfplatte reichend. Vorn an der Basis der Säume jederseits eine Gruppe kleiner Ocellen (Abb. 7B). Vordere 5 Borstensegmente kurz, nur wenig länger als breit; in den vorderen 4 Segmenten die Borsten etwas näher am Vorderrand, im 5. etwa in der Mitte entspringend. Vorderrand der Borstensegmente 2 bis 4 schwach kragenartig. Vorderer Abschnitt des 4 . und 5. Borstensegments etwas angeschwollen und drüsig; hinterer Abschnitt des 1.-5. Borstensegments leicht geringelt (Abb. 7B). 6. Borstensegment nur wenig länger als das 5.; Borsten in der Mitte. Abschnitt vor den Borsten dick und drüsig, hinter den Borsten geringelt und undeutlich rötlich gefärbt. 7 . Borstensegment fast doppelt so lang wie das 6.; Borsten hinter dem vorderen Drittel. Abschnitt vor den Borsten hell und grob und unregelmäßig geringelt; hinter den Borsten ebenso geringelt und schwach rötlich: über den ganzen Rücken und ventral in Form eines schmalen Längsbandes. 8. Borstensegment unvollständig, anscheinend ähnlich wie das 7.; Borsten eventuell in der Mitte. 


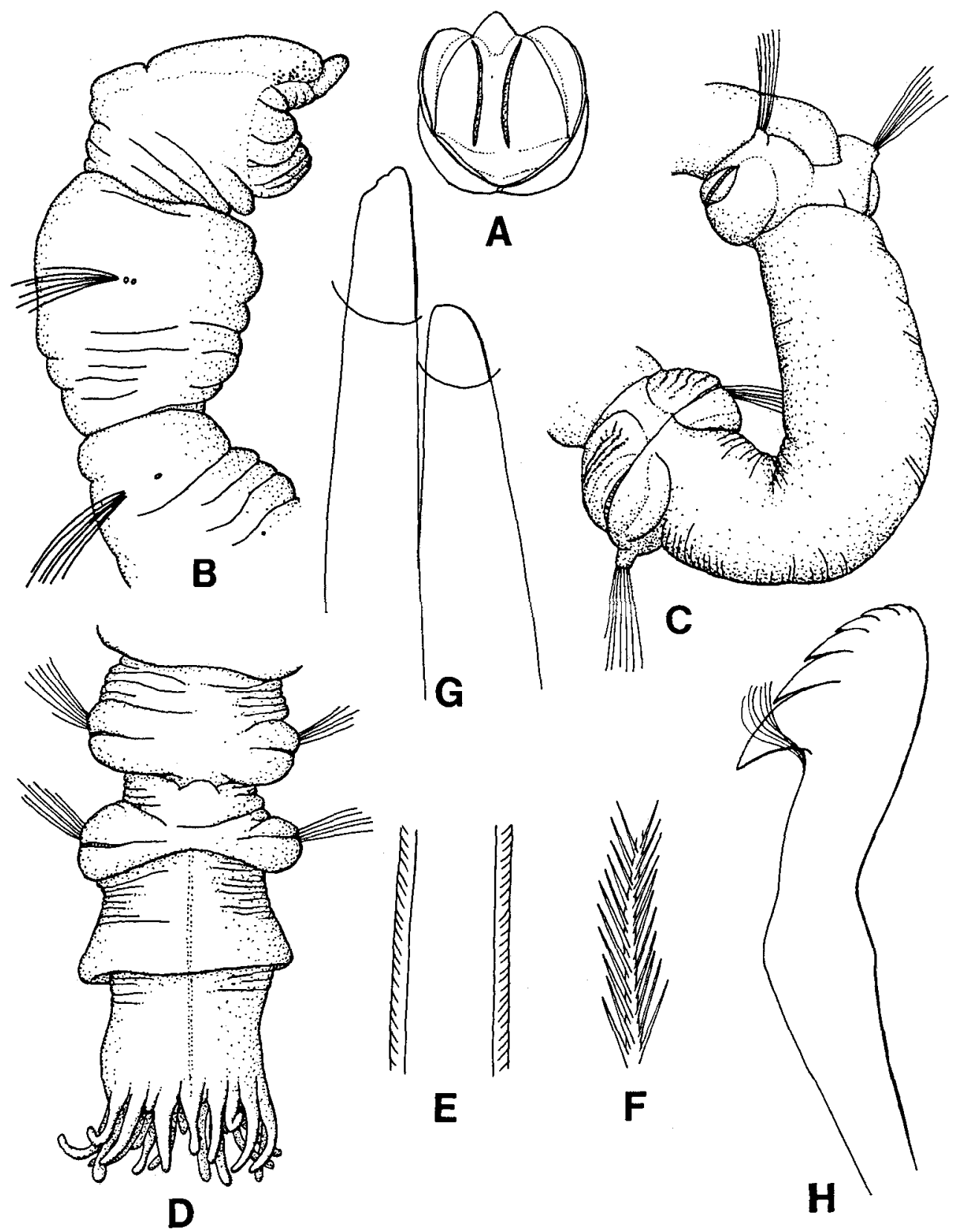

Abb.7. Euclymene (E.) sp. A: Kopfplatte. B: Vorderende von lateral. C: mittlere Segmente. D: Hinterende von ventral. E: Teil einer gesäumten Borste. F: Teil einer gefiederten Borste. G: Haken aus 1. Neuropod. H: Haken aus 8. Neuropod 
2 anscheinend hierauf folgende Segmente (9. und 10.) etwa $11 / 2 \mathrm{mal}$ so lang wie breit, besonders das vordere dorsal rötlich.

Vorderes Segment des vorderen mittleren Bruchstücks etwa 5 mal so lang wie breit; Borsten und Haken auf Wuist am Finterrand. Vorderrand des Wulstes weißlich-drüsig; hinter den Haken ein weißliches Drüsenpolster, das in dreieckigem Zipfel bis zur hinteren Basis des Notopods reicht; Polster dahinter mit 3-4 unregelmäßig gewellten hellbraunen Furchen (Abb. $7 \mathrm{C}$ ). Folgende 5 Segmente ähnlich, jedoch langsam kürzer werdend; das hintere nur noch wenig mehr als doppelt so lang wie breit.

Vorderes Segment des hinteren mittleren Bruchstücks so wie letztes Segment des vorderen mittleren Bruchstücks, etwa doppelt so lang wie breit. Folgende 4 Segmente sowie die vorderen 2 des Hinterendes sehr kurz, etwas breiter als lang, kampanulat; Wülste ähnlich wie bei den Segmenten des vorderen mittleren Bruchstücks, die bräunlichen Furchen allmählich schwächer werdend. Wülste wie dort am Hünterrand. Auch letzte Borstensegmente des Hinterendes kurz, etwas breiter als lang. Präanales borstenloses Segment nach hinten kragenartig. Analtrichter mit 19 längeren und 5 sehr unterschiedlich kurzen Analcirren; keine längere medio-ventrale Cirre (Abb. 7D). Anus am Grunde des Trichters auf kleinem radiär gefältetem Kegel.

Borsten von 2 Sorten: (1) breitere, längere, gesäumte Kapillarborsten und (2) wenige dünnere, fein gefiederte Borsten (Abb. 7E-F). Vordere 3 Paar Neuropodien mit jeweils 2 (nur im 2. rechten Neuropod 1) aciculären Haken (Abb. 7G). Folgende Neuropodien mit "normalen" Haken, mit 6 Zähnchen in einer senkrechten Reihe über dem Hauptzahn; dazu jederseits ein Bündel gebogener Rostralhaare (Abb. $7 \mathrm{H}$ ). In einem mittleren Neuropod 24 Haken vorhanden.

Fundort: Bahia Quillaipe: Sandwatt nahe der Niedrigwasserlinie; 09. 01.89: 1 Exemplar und 1 mittleres Bruchstück sowie 1 Hinterende.

T axo nomische Bemerkung: Da nicht absolut sicher ist, ob die beschriebenen Einzelteile wirklich so und lückenlos zusammengehören, soll die Art nicht benannt werden. Für den Fall, daß alles so fehlerlos zu einem Tier zusammenpaßt, wäre es eine neue Art.

Am ähnlichsten sind 3 Arten: E. grossa Baird, E. lüderitziana Augener und E. natalensis (Day). E. grossa und E. natalensis besitzen nur 19 Borstensegmente. Die Form der Kopfplatte mit den Ocellen und der Haken stimmen gut mit den Verhältnissen beí $E$. natalensis überein; dort sind jedoch 2 präanale borstenlose Segmente vorhanden, die hinteren Borstensegmente sind viel länger, und auch die medio-ventrale Analcirre ist länger als die übrigen. E. grossa hat wie die vorliegende Art nur 1 präanales borstenloses Segment; ihr fehlen jedoch Augenflecke, und der hintere Saum der Kopfplatte ist fein gekerbt. E. lüderitziana stimmt überein in der Zahl der Borstensegmente (23-24) und in der Form der Kopfplatte mit den Ocellen. Es sind bei ihr jedoch 2 präanale borstenlose Segmente entwickelt, und die letzten Borstensegmente sind etwas mehr als doppelt so lang wie breit; außerdem ist eine medio-ventrale Analcirre länger als die übrigen Analcirren.

Familie Arenicolidae Mesnil, 1898

Gattung Abarenicola Wells, 1959

Abarenicola affinis chilensis Wells, 1963 
1954 Arenicola assimilis var. affinis, - Wells: 3.

1963a Abarenicola affinis chilensis Wells: 143.

1963b Abarenicola affinis chilensis, - Wells: 88.

Ein Tier mit 19 Borstensegmenten und borstenlosem Schwanz etwa $110 \mathrm{~mm}$ lang. Färbung in Alkohol dunkel bräunlich-violett-schwärzlich; nach Reise lebendes Tier schwarz-braun mit grünlichem Schimmer, Kiemen dunkelbraun, Pharynx braun-rot. Kiemen $a b$ 7. Borstensegment. Zwischen 1. und 2. borstentragendem Ring 1 breiter und 1 schmaler Ring; desgleichen zwischen 2. und 3. borstentragendem Ring (nach Wells 1963a, nur 1; hier der letzte aber sehr schmal); zwischen 3. und 4. borstentragendem Ring 4 Ringe vorhanden. Dieses Muster trifft auf keine andere Abarenicola-Art zu, die auch in Chile vorkommt.

Fund ort: Bahia Quillaipe: Schlickwatt mit Sand; 15. 01. 89: 1 Exemplar.

Verbreitung: Süd-Chile.

Ökologie : Substrat: Sand, Schlick-Sand; Tiefe: Eulitoral.

Familie Terebellidae Malmgren, 1865

Unterfamilie Polycirrinae Hessle, 1917

Gattung Polycirrus Grube, 1850

\section{Polycirrus sp.}

Größtes der nicht gut erhaltenen Tiere mit 17 oder 18 Borstensegmenten im Thorax und zahlreichen Abdominalsegmenten $22 \mathrm{~mm}$ lang. Lebendfärbung: Thorax rot, Abdomen gelblich. Borsten teilweise fein gefiedert. Haken ab 1. Abdominalsegment. Röhren aus Sand.

F u n d or t: Bahia Quillaipe: Sandwatt; 15. 01. 89: 6 Exemplare,

\section{ZOOGEOGRAPHIE}

Von den 15 bekannten und bestimmbaren Arten sind 4 Arten weit verbreitet. 4 Arten haben ihr Verbreitungsgebiet im südlichen Südamerika, in der Subantarktis und Antarktis sowie ( 3 von ihnen) in Australien und Neuseeland. Eine Art ist bisher nur vom südlichen Australien (Tasmanien) und von Chile bekannt geworden. 6 Arten schließlich kommen nur an den Kaltwasserküsten Südamerikas vor: 4 Arten nur im Gebiet des Humboldt-Stroms und 2 sowohl im Humboldt-Strom als auch im Falkland-Strom-Gebiet (Ost-Patagonien). Exogone obtusa tasmanica, Lumbrineris patagonica und Spiochaetopterus costarum wurden erstmals in Chile nachgewiesen.

\section{LITERATUR}

Augener, H., 1926. Polychaeten von Neuseeland. II. Sedentaria. - Vidensk. Meddr dansk. naturh. Foren. 81, 157-294.

Blake, J. A., 1983. Polychaetes of the family Spionidae from South America, Antarctica, and adjacent seas and islands. - Antarct. Res. Ser. 39, 205-288.

Carrasco, F. D., 1974. Spionidae (Polychaeta) provenientes de la Bahia de Concepción y lugares adyacentes. - Bol. Soc. biol. Concepción 48, 185-201. 
Carrasco, F. D., 1975. Ciclo de la presencia de larvas de Polychaeta (Annelida) en el plancton de la Bahía de Concepción, Chile. - Investnes oceanol. chil. 2, 3-12.

Carrasco, F. D., 1976. Larvas de la familia Spionidae (Polychaeta) en el plancton de la Bahía de Concepción, Chile. - Gayana, Zool. 38, 3-63.

Claparède, E., 1868. Les Annélides Chétopodes du Golfe de Naples. - Mem. Soc. Phys. Hist. nat. Genève 19, 313-584.

Claparède, E., 1870. Les Annélides Chétopodes du Golfe de Naples. - Mem. Soc. Phys. Hist. nat. Genève 20, 1-225, 365-542.

Ehlers, E., 1897. Polychaeten. - Hamburger Magalhaenische Sammelreise. Friedrichsen, Hamburg, $148 \mathrm{pp}$.

Ehlers, E., 1900. Magellanische Anneliden, gesammelt während der schwedischen Expedition nach den Magellansländern. - Nachr. Ges. Wiss. Göttingen 1900, 206-223.

Ehlers, E., 1901a. Die Polychaeten des magellanischen und chilenischen Strandes. Ein faunistischer Versuch. - Festschr. Feier 150jähr. Besteh. K. Ges. Wiss. Göttingen. Wiedemann, Berlin, 232 pp.

Ehlers, E., 1901b. Die Anneliden der Sammlung Plate. Fauna Chilensis. - Zool. Jb. (Suppl.) 5, 251-272.

Gilbert, K. M., 1984. Family Chaetopteridae Malmgren, 1867. In: Taxonomic guide to the polychaetes of the northern Gulf of Mexico. Ed. by J. M. Uebelacker. Vittor, Alabama, 2, 11.1.-11.13.

Gitay, A., 1969. A contribution to the revision of Spiochaetopterus (Chaetopteridae, Polychaeta). Sarsia 37, 9-20.

Grube, A. E., 1857. Annulata Oerstediana, Enumeration Annulatorum, qua in itinere per Indiam occidentalem et Americam centralem annis 1845-1848 suspecto legit cl. A. S. Oersted, adjectis speciebus nonnullis a. cl. H. Kröyer in itinere ad Americam meridionalem collectis. 2, 158-166.

Hartman, O., 1940. Polychaetous annelids. Pt. 2. Chrysopetalidae to Goniadidae. - Allan Hancock Pacif. Exped. 7, 173-287.

Hartman, O., 1953. Non-pelagic Polychaeta of the Swedish Antarctic Expedition 1901-1903. Further zool. Results Swed. Antarc. Exped. 4 (11), 1-83.

Hartman, O, 1964. Polychaeta Errantia of Antarctica. - Antarc. Res. Ser. 3, 1-131.

Hartman, O., 1966. Polychaeta Sedentaria and Myzostomida of Antarctica. - Antarct. Res. Ser. 7, $1-158$.

Hartman, O., 1967. Polychaetous annelids collected by the USNS ELTANIN and STATEN ISLAND CRUISES, chiefly from Antarctic seas. - Allen Hancock Monogr. mar. Biol. 2, 1-387.

Hartmann-Schröder, G., 1962a. Zur Kenntnis des Eulitorals der chilenischen Pazifikküste und der argentinischen Küste Südpatagoniens unter besonderer Berücksichtigung der Polychaeten und Ostracoden. Teil II: Die Polychaeten des Eulitorals. - Mitt. hamb. zool. Mus. Inst. 60 (Erg.Bd), 57-167.

Hartmann-Schröder, G., 1962b. Zur Kenntnis der Nereiden Chiles (Polychaeta Errantia), mit Beschreibung epitoker Stadien einiger Arten und der Jugendentwicklung von Perinereis vallata (Grube). - Zool. Anz, 168, 389-441.

Hartmann-Schröder, G, 1965. Zur Kenntnis des Sublitorals der chilenischen Küste unter besonderer Berücksichtigung der Polychaeten und Ostracoden. Teil II: Die Polychaeten des Sublitorals. Mitt. hamb. zool. Mus. Inst. 62 (Erg.Bd), 59-305.

Hartmann-Schröder, G., 1980. Zur Kenntnis des Eulitorals der australischen Küsten unter besonderer Berücksichtigung der Polychaeten und Ostracoden. Teil 4: Die Polychaeten der tropischen Nordwestküste Australiens (zwischen Port Samson im Norden und Exmouth im Süden). - Mitt. hamb. zool. Mus. Inst. 77, 41-110.

Hartmann-Schröder, G., 1989. Zur Kenntnis des Eulitorals der australischen Küsten unter besonderer Berücksichtigung der Polychaeten und Ostracoden. Teil 14: Die Polychaeten der antiborealen und subtropisch-tropischen Küste Südost-Australiens zwischen Lakes Entrance (Victoria) im Süden und Maclean (New South Wales) im Norden. - Mitt. hamb. zool. Mus. Inst. 86, 11-63.

Haswell, W. A., 1885. On a destructive parasite of a rock oyster (Polydora ciliata and P. polybranchia n. sp.). - Proc. Linn. Soc. N.S.W., 10, 272-275.

Hutchings, P. A. \& Turvey, S. P., 1984. The Spionidae of South Australia (Annelida: Polychaeta), Trans. R. Soc. S. Aust. 108, 1-20.

Kinberg, J. G. H., 1866. Annulata nova. - Oefv. Vet. Akad. Stockholm, Forh. 22, 167-179; $239-258$. 
Orensanz, J. M., 1974. Los anélidos poliquetos de la Provincia biogeográfica Magellánica I. Contrnes. técn. Lab. Comunid. Benton. 1, 3-76.

Ringuelet, R. A., 1969. Clave o llave para el reconocimiento de familias y géneros de poliquetos del litoral Atlántíco Argentino. - Act. zool. lilloana 24, 193-218.

Rozbaczylo, N. \& Castilla, J. C., 1973. El género Perinereis (Annelida, Polychaeta, Nereidae) en Chile. - Stud. neotrop. Fauna Environm. 8, 215-232.

Rozbaczylo, N. \& Castilla, J. C., 1974. La familia Nephtyidae en Chile (Annelida, Polychaeta). - Stud. neotrop. Fauna Environm. 9, 179-206.

Schmarda, L. K., 1861. Neue wirbellose Tiere beobachtet und gesammelt auf einer Reise um die Erde 1853 bis 1857. Leipzig, 1, 1-164.

Wells, G. P. A., 1954. A preliminary account of the Arenicolidae. Reports of the Lund Univ. Chile Expedition 1948-49. - Lunds Univ. Arsskr. (N.F.Avd.2) 50 (8), 1-6.

Wells, G. P. A., 1963a. The lugworms of the southern cold temperature zone (Arenicolidae, Polychaeta). - Proc. zool. Soc. Lond 140, 121-159.

Wells, G. P. A., 1963b. Barriers and speciation in lugworms (Arenicolidae, Polychaeta). - Syst. Ass. Publ. 5, 79-98.

Wesenberg-Lund, E., 1961. Polychaeta Errantia. Reports of the Lund Univ. Chile Expedition 1948-49. - Lunds Univ. Arsskr. (N.F.Avd.2) 57 (12), 1-137. 